\title{
Prognostic Factors Influencing Clinical Outcomes of Malignant Glioblastoma Multiforme: Clinical, Immunophenotypic, and Fluorescence in Situ Hybridization Findings for 1p19q in 816 Chinese Cases
}

\author{
Jun-Jie Qin ${ }^{1}$, Zhao-Xia Liu ${ }^{1}$, Jun-Mei Wang ${ }^{1}$, Jiang Du${ }^{1}$, Li Xu${ }^{1}$, Chun Zeng ${ }^{2}$, \\ Wu Han ${ }^{2}$, Zhi-Dong $\mathrm{Li}^{2}$, Jian Xie ${ }^{2 *}$, Gui-Lin $\mathrm{Li}^{1 *}$
}

\begin{abstract}
Malignant glioblastoma multiforme (GBM) is the most malignant brain tumor and despite recent advances in diagnostics and treatment prognosis remains poor. In this retrospective study, we assessed the clinical and radiological parameters, as well as fluorescence in situ hybridization (FISH) of 1p19q deletion, in a series of cases. A total of 816 patients with GBM who received surgery and radiation between January 2010 and May 2014 were included in this study. Kaplan-Meier survival analysis and Cox regression analysis were used to find the factors independently influencing patient progression free survival (PFS) and overall survival (OS). Age at diagnosis, preoperative Karnofsky Performance Scale (KPS) score, KPS score change at 2 weeks after operation, neurological deficit symptoms, tumor resection extent, maximal tumor diameter, involvement of eloquent cortex or deep structure, involvement of brain lobe, Ki-67 and MMP9 expression level and adjuvant chemotherapy were statistically significant factors $(p<0.05)$ for both PFS and OS in the univariate analysis. Cox proportional hazards modeling revealed that age $\leq 50$ years, preoperative KPS score $\geq 80$, KPS score change after operation $\geq 0$, involvement of single frontal lobe, deep structure involvement, low Ki-67 and MMP9 expression and adjuvant chemotherapy were independent favorable factors $(\mathbf{p}<0.05)$ for patient clinical outcomes.
\end{abstract}

Keywords: Malignant glioblastoma multiform - prognostic factor - FISH of 1p19q - immunohistochemistry

Asian Pac J Cancer Prev, 16 (3), 971-977

\section{Introduction}

Malignant glioblastoma multiform (GBM, WHO grade IV) are the most malignant and prevalent intracranial tumor providing for well over one half of all gliomas (Hoffman et al., 2006; Schneider et al., 2010). GBM is characterized by a rapid growth and short time to progression in most treated patients. Current therapeutic modalities include surgical resection, radio-surgical or external irradiation, chemotherapy (Cheng et al., 2005; Stupp et al., 2006; Wen et al., 2006) and biological therapy (Kjaergaard et al., 2005). Despite recent advances in diagnostics and treatments, prognosis for advanced patients suffering from these diseases remains poor $(\mathrm{Ng}$ et al., 2007). The median survival time is usually less than a year (Sathornsumetee and Rich, 2006). It is important for us to understand the factors that contribute positively or negatively to the prognosis of patients, which may guide treatment paradigms and therapeutic strategies aimed at prolonging survival. Some researchers have analyzed the role of patients' clinical characteristics and tumor imaging features (Jeremic et al., 2003; Kang et al., 2005; Tramacere et al., 2008). Tumor molecular alteration has been taken into consideration in few studies (Gorlia et al., 2008; Li et al., 2008). Few studies are based on a large scale of Chinese GBM patients.

In this retrospective study, we analyzed a large institutional series of Chinese patients with GBM to identify clinical., radiological., Immunohistochemistry and treatment factors influencing the clinical outcomes.

\section{Materials and Methods}

\section{Patient selection}

1186 cases of malignant glioblastoma (stage IV tumors) were collected from the Neuropathology Department of the Beijing Neurosurgical Institute and Beijing Tiantan hospital from January 2010 to May 2014. 373 cases of them were excluded because of insufficient materials or incomplete clinical data. The remaining 816

${ }^{1}$ Department of Neuropathology, Beijing Neurosurgical Institute, ${ }^{2}$ Department of Neurosurgery, Beijing Tiantan Hospital, Capital Medical University; China National Clinical Research Center for Neurological Diseases; Center of Brain Tumor, Beijing Institute for Brain Disorders; Beijing Key Laboratory of Brain Tumor; Beijing, China *For correspondence: liguilin2014@126.com, xiejianw@sina.com 
$(68.80 \%)$ cases were included in this study. Histologic diagnosis of the tumors was based on the World Health Organization classification of tumors of Nervous System (Louis et al., 2007).

The following data were collected from the medical records of the patients: (i) Examination of histologic features on slides of GBM tissues; (ii) Immunophenotype analysis by immunohistochemistry, which performed in 306 cases, including MMP-9; PTEN; EGFR; P53; VEGF; Ki-67; TOPOII; GST- $\pi$. The staging evaluation was based on the Ann Arbor system (1971). FISH of 1p19q were performed in 80 cases. (iii) Demographic data of these patients (age and gender), Preoperative Karnofsky Performance Scale (KPS) scores and KPS scores at two weeks after operation. (iv) Follow-up data were obtained for the all patients: clinical outcomes including progression free survival (PFS) and overall survival (OS) time, which were mainly collected when patients visited the clinics or during phone interviews with patients and/or their relatives. $(v)$ All patients had a staging evaluation that consisted of magnetic resonance imaging (MRI) scans of the neuro system. Preoperative and postoperative contrast enhancing magnetic resonance imaging (MRI): tumor radiological characteristics (including surrounding edema, cyst formation, contrast enhancement, maximal diameter, midline shift, contralateral involvement, hemisphere involvement, involvement of eloquent cortex or deep structure and involvement of brain lobe) assessed by a neuroradiologist based on preoperative MRI, extent of resection assessed on the postoperative enhanced MR image within 24 hours and graded as total or subtotal resection.

\section{Immunohistochemistry}

Immunohistochemistry was performed in 292 $(35.78 \%)$ cases. The following antibodies were used: matrix metallopeptidase 9 (MMP-9) (polyclonal; ZSGB-BIO);phosphatase and tension homology deleted onchromosome ten (PTEN) (28H6; DAKO);epidermal growth factor receptor (EGFR) (EP22; DAKO);P53 (BP 53.12; ZSGB-BIO, Beijing, China); Ki-67 (MIB-1; DAKO); TOPOII (3F6; DAKO);GST- $\pi$ (LW29; ZSGBBIO, Beijing, China). A series of 4- $\mu$ m paraffin sections were deparaffinized in xylene and hydrated in a graded series of ethanol. All the slides were treated in EDTA (1 mmol/L, $\mathrm{pH}$ 9.0) for 8 minutes. The EnVision method (DAKO) was used for immunohistochemical staining, with diaminobenzidine as substrate; PBS was used instead of first antibody as negative control. Positive results exhibit brown yellow granules attached respectively to cell membrane, cytoplasm or nucleus. Negative results are no coloring in cell membrane, cytoplasm, or nucleus. In case of a discrepancy, the two observers simultaneously reviewed the slides to achieve a consensus. The expression of individual molecule was graded as high or low for the analysis.

\section{FISH of $1 p 19 q$ deletion}

FISH data were obtained for $80(9.80 \%)$ of the 816 patients, most of them were GBM contain oligodendroglioma component. FISH was conducted using the FISH pharmDx ${ }^{\mathrm{TM}}$ (Dako Denmark A/S, Glostrup, Denmark). The assay was performed according to the manufacturer's recommendations and subsequently evaluated using a fluorescence microscope equipped with appropriate filters for 4'-6-diamidino-2-phenylindole, fluorescein isothiocyanate (FITC) and Texas Red at $60 \times$ or $100 \times$ magnifications.

\section{Treatment}

Standard treatment was consisted of surgery and postoperative radiotherapy, with or without adjuvant chemotherapy. Maximal tumor bulk resection while preserving the key eloquent cortex was the principle of operation. Preoperative functional MRI and awaking mapping were used if necessary. Postoperative limitedradiotherapy was routinely delivered to the patient within 1 month after surgery. The total dose was $60 \mathrm{~Gy}$, which was

Table 1. Clinical and Treatment Factors Associated with PFS and OS in the Univariate Analysis

\begin{tabular}{|c|c|c|c|c|c|c|}
\hline Variable & & Cases(n) & Median PFS (days) & $\mathrm{p}$ values & Median OS (days) & $\mathrm{p}$ values \\
\hline \multirow[t]{2}{*}{ Gender } & Male & 470 & 254 & 0.11 & 508 & 0.29 \\
\hline & Female & 336 & 338 & & 604 & \\
\hline \multirow[t]{2}{*}{ Age(year) } & $\leq 45$ & 605 & 337 & 0.03 & 565 & 0.01 \\
\hline & $>45$ & 211 & 181 & & 331 & \\
\hline \multirow[t]{2}{*}{ Headache } & Yes & 613 & 257 & 0.07 & 508 & 0.64 \\
\hline & No & 203 & 229 & & 449 & \\
\hline \multirow[t]{2}{*}{ Seizure } & Yes & 418 & 259 & 0.74 & 449 & 0.67 \\
\hline & No & 398 & 254 & & 508 & \\
\hline \multirow[t]{2}{*}{ Neurological deficit symptoms } & Yes & 298 & 164 & 0.00 & 283 & 0.01 \\
\hline & No & 518 & 338 & & 565 & \\
\hline \multirow[t]{2}{*}{ Preoperative KPS score } & $\geq 80$ & 610 & 349 & 0.00 & 601 & 0.00 \\
\hline & $<80$ & 206 & 184 & & 319 & \\
\hline \multirow[t]{2}{*}{ KPS score change at 2 weeks after operation } & $\geq 0$ & 557 & 339 & 0.00 & 565 & 0.00 \\
\hline & $<0$ & 259 & 119 & & 216 & \\
\hline \multirow[t]{2}{*}{ GBM type } & Primary & 748 & 295 & 0.59 & 554 & 0.23 \\
\hline & Secondary & 68 & 245 & & 328 & \\
\hline \multirow[t]{2}{*}{ Extent of resection } & Gross total & 209 & 383 & 0.01 & 604 & 0.04 \\
\hline & Reduction & 607 & 242 & & 449 & \\
\hline \multirow[t]{2}{*}{ Adjuvant chemotherapy } & Yes & 466 & 356 & 0.01 & 604 & 0.00 \\
\hline & No & 350 & 234 & & 331 & \\
\hline
\end{tabular}

*PFS: progression free survival; OS: overall survival; KPS: karnofsky performance scale; GBM: glioblastoma multiforme 
Prognostic Factors Influencing Clinical Outcomes of Malignant Glioblastoma Multiforme

divided into 30 daily fractions of 2 Gy each. For patients receiving adjuvant chemotherapy, the treatment was given 1 month after radiation and composed of at least two cycles of chemotherapy. Combined drugs were used and made according to the drug-resistant protein expression status. A total of six cycles were to be administered if no disease progression occurred and there were no irreversible hematological toxic effects.

\section{Statistical analysis}

Our primary goals were to reveal which parameters were associated with the clinical outcomes of the patients. Tumor progression was defined according to the modified WHO criteria (Macdonald et al., 1990), as an increase of tumor size by $25 \%$ or appearance of new lesions. All timing was referenced to the date of operation, e.g. PFS time as the interval between operation and radiographic progression, OS time as the period from operation to death. Survivor function curves were calculated with the Kaplan-Meier method and differences were evaluated with the log-rank test. Multivariate Cox models were used after univariate analysis. SPSS 13.0 software for Windows (Chicago, IL) was used for statistical analysis Overall survival time was estimated from the date of diagnosis to the date of death or last follow-up and was plotted using the Kaplan-Meier method. A P value $<0.05$ were considered as statistically significant.

\section{Results}

\section{General data}

Totally 816 patients were recruited in the study, including 748 primary and 68 secondary GBM. Of the 816 cases, 209 had total tumor resection and 607 had subtotal

Table 2. Radiological and Genetic Factors Associated with PFS and OS in the Univariate Analysis

\begin{tabular}{|c|c|c|c|c|c|c|c|}
\hline \multicolumn{3}{|l|}{ Variable } & Cases(n) & Median PFS (days) & $\mathrm{p}$ values & Median OS (days) & $\mathrm{p}$ values \\
\hline \multirow[t]{2}{*}{ Surrounding edema } & \multicolumn{2}{|l|}{ Yes } & 438 & 245 & 0.37 & 464 & 0.62 \\
\hline & \multicolumn{2}{|l|}{ No } & 378 & 350 & & 604 & \\
\hline \multirow[t]{2}{*}{ Cyst formation } & \multicolumn{2}{|l|}{ Yes } & 431 & 339 & 0.19 & 508 & 0.33 \\
\hline & \multicolumn{2}{|l|}{ No } & 385 & 240 & & 565 & \\
\hline \multirow[t]{2}{*}{ Contrast enhancement } & \multicolumn{2}{|l|}{ Yes } & 502 & 259 & 0.34 & 508 & 0.42 \\
\hline & \multicolumn{2}{|l|}{ No } & 314 & 236 & & 532 & \\
\hline \multirow[t]{2}{*}{ Midline shift } & \multicolumn{2}{|l|}{ Yes } & 489 & 249 & 0.33 & 464 & 0.43 \\
\hline & \multicolumn{2}{|l|}{ No } & 327 & 340 & & 604 & \\
\hline \multirow[t]{2}{*}{ Contralateral involvement } & \multicolumn{2}{|l|}{ Yes } & 313 & 298 & 0.14 & 335 & 0.12 \\
\hline & \multicolumn{2}{|l|}{ No } & 503 & 328 & & 565 & \\
\hline \multirow[t]{2}{*}{ Maximal diameter } & \multicolumn{2}{|l|}{$<5 \mathrm{~cm}$} & 474 & 339 & 0.03 & 565 & 0.03 \\
\hline & \multicolumn{2}{|l|}{$\geq 5 \mathrm{~cm}$} & 342 & 250 & & 442 & \\
\hline \multirow[t]{2}{*}{ Hemisphere involvement } & \multicolumn{2}{|l|}{ Left } & 341 & 259 & 0.8 & 565 & 0.91 \\
\hline & \multicolumn{2}{|l|}{ Right } & 475 & 338 & & 508 & \\
\hline \multicolumn{8}{|c|}{ Eloquent cortex or deep structure involvement } \\
\hline & Yes & & 454 & 181 & 0 & 331 & 0 \\
\hline & No & & 362 & 383 & & 604 & \\
\hline Involvement of brain lobe & Single frontal lobe & & 315 & 567 & 0 & 203 & 0.01 \\
\hline & Other single lobe & & 194 & 349 & & 60 & \\
\hline & Multiple lobe & & 307 & 185 & & 406 & \\
\hline Ki-67 & Low & & 66 & 338 & 0.01 & 604 & 0.03 \\
\hline & High & & 226 & 201 & & 449 & \\
\hline PTEN & Low & & 73 & 274 & 0.36 & 449 & 0.88 \\
\hline & High & & 193 & 250 & & 508 & \\
\hline MMP-9 & Low & & 66 & 305 & 0.03 & 464 & 0.03 \\
\hline & High & & 179 & 259 & & 508 & \\
\hline EGFR & Low & & 36 & 340 & 0.24 & 565 & 0.12 \\
\hline & High & & 207 & 259 & & 508 & \\
\hline TOPOII & Low & & 158 & 234 & 0.632 & 533 & 0.134 \\
\hline & High & & 134 & 321 & & 487 & \\
\hline GST- $\pi$ & Low & & 104 & 335 & 0.302 & 564 & 0.67 \\
\hline & High & & 150 & 267 & & 532 & \\
\hline VEGF & Low & & 123 & 206 & 0.143 & 488 & 0.899 \\
\hline & High & & 134 & 276 & & 498 & \\
\hline P53 & Low & & 126 & 355 & 0.053 & 498 & 0.069 \\
\hline & High & & 146 & 206 & & 398 & \\
\hline FISH of $1 p 19 q$ & 1p36 deletion & + & 10 & 332 & 0.321 & 456 & 0.899 \\
\hline & & - & 70 & 258 & & 567 & \\
\hline & & polyploidy & 15 & 245 & & 512 & \\
\hline & $19 \mathrm{q} 13$ deletion & + & 5 & 289 & 0.645 & 578 & 0.345 \\
\hline & & - & 75 & 206 & & 543 & \\
\hline & & polyploidy & 23 & 276 & & 489 & \\
\hline & 1p19q codeletion & + & 2 & 334 & 0.213 & 508 & 0.564 \\
\hline & & - & 78 & 267 & & 486 & \\
\hline
\end{tabular}


Table 3. Prognostic Factors Associated with PFS and OS in the Multivariate Analysis

\begin{tabular}{|c|c|c|c|c|}
\hline \multicolumn{2}{|c|}{ Variable } & \multirow{2}{*}{$\frac{\text { Hazard ratio }}{1.83}$} & \multirow{2}{*}{$\frac{95 \% \mathrm{CI}}{0.98-3.54}$} & \multirow{2}{*}{$\frac{p \text { values }}{0.03}$} \\
\hline PFS & Age & & & \\
\hline & Involvement of brain lobe & 1.59 & $1.21-2.09$ & 0.01 \\
\hline & Preoperative KPS score & 0.53 & $0.28-0.93$ & 0.01 \\
\hline & KPS score change at 2 weeks after operation & 0.28 & $0.09-0.47$ & 0.01 \\
\hline & Ki-67 & 3.55 & $1.47-6.31$ & 0.01 \\
\hline & MMP9 & 2.13 & $0.98-4.36$ & 0.02 \\
\hline & Adjuvant chemotherapy & 0.31 & $0.14-0.59$ & 0.01 \\
\hline \multirow[t]{7}{*}{ OS } & Age & 3.27 & $1.21-5.76$ & 0.01 \\
\hline & Eloquent cortex or deep structure involvement & 2.43 & $1.22-4.19$ & 0.01 \\
\hline & Preoperative KPS score & 0.31 & $0.14-0.59$ & 0 \\
\hline & KPS score change at 2 weeks after operation & 0.45 & $0.10-0.74$ & 0.01 \\
\hline & Ki-67 & 4.33 & $1.24-7.76$ & 0.01 \\
\hline & MMP9 & 3.1 & $1.28-5.36$ & 0.04 \\
\hline & Adjuvant chemotherapy & 0.53 & $0.25-0.93$ & 0.04 \\
\hline
\end{tabular}

tumor resection. For treatment, 466 patients had surgery, radiation and adjuvant chemotherapy and the other 350 patients had surgery and radiation only. In a median follow-up of 14 months (range: 4-30 months), 76 cases Alive with disease, 206 cases suffered tumor progression and 534 cases died. The patients' median PFS time was 274 days (95\% CI: 256-296 days) and median OS time was 508 days (95\% CI: 444-474 days).

\section{Univariate analysis}

According to the univariate analysis, clinical factors associated with PFS and OS are reported in Table 1. Age at diagnosis, preoperative KPS score, KPS score change at 2 weeks after operation and neurological deficit symptoms were statistically significant factors for PFS and OS. Both tumor resection extent and adjuvant chemotherapy were correlated with PFS and OS. Radiological and molecular factors associated with PFS and OS are showed in Table 2. Tumor maximal diameter, involvement of eloquent cortex or deep structure and involvement of brain lobe were radiological significant factors related to PFS and OS. Generous molecular changes were found in the GBM patients. Ki-67 and MMP9 was the molecule statistically related to the patients' PFS and OS. FISH of 1p19q were not correlated with PFS and OS.

\section{Multivariate analysis}

Factors with corresponding $p$ values $<0.05$ in the univariate analysis were introduced into multivariate model. Age at diagnosis $(p=0.03)$, preoperative KPS score $(p=0.01)$, KPS score change after operation $(p=0.01)$, involvement of brain lobe $(p=0.01)$, Ki-67 and MMP9 expression level ( $p=0.01$ and 0.02$)$ and adjuvant chemotherapy ( $p=0.01$ ) were independent factors for PFS. In regards to OS, age at diagnosis $(p=0.01)$, preoperative KPS score $(p=0.00)$, KPS score change after operation $(p=0.01)$, involvement of eloquent cortex or deep structure $(p=0.01), \mathrm{Ki}-67$ and MMP9 expression level ( $p=0.01$ and $0.004)$ and adjuvant chemotherapy $(p=0.04)$ persisted as statistically independent prognostic factors. Among them, age $\leq 50$, preoperative KPS $\geq 80$, KPS score change after operation $\geq 0$, involvement of single frontal lobe, noneloquent area or deep structure involvement, low Ki-67 and MMP9 expression and adjuvant chemotherapy were favorable factors (Table 3).

\section{Discussion}

Astrocytomas are primary brain tumors of glial lineage and these are classified by the World Health Organization (WHO) into four grades (Louis et al., 2007). Glioblastoma multiform (GBM or grade IV astrocytoma), or simply glioblastoma, represents the most aggressive and malignant entity. Pathological features of GBM comprise the presence of atypia, pleomorphism, mitoses, pseudopalisading cells, and central necrosis together with a high and abnormal vascularisation. Two types of GBMs are classically distinguished: 'primary GBMs', which develop de novo in previously healthy individuals, and 'secondary GBMs', which represent the malignant progression of a lower-grade astrocytoma (typically grade I or grade III). In particular, primary glioblastomas are characterized by EGFR amplification, loss of PTEN; while secondary glioblastomas present p53 mutations and abnormal activation of PDGFRa. In general., uncontrolled local recurrence and dissemination along the white matter tracts represent the final stage of the disease while metastases out of the central nervous system (CNS) have exceptionally been reported. The marked invasiveness is in fact considered a hallmark of GBMs, which infiltrate the normal brain parenchyma well beyond what can be seen on the neuroradiological investigations. Despite surgical debulking, radiation, and chemotherapy, prognosis of glioblastoma remains poor with a mean survival of less than 16 months (Stupp et al., 2005; Stupp and Weber, 2005). The prognosis depends on several factors including age of the patient, Karnofsky performance score (KPS), size, and site of the tumor.

For the dismal clinical outcomes of GBM, a full-scale knowledge of the prognostic factors from a large quantity of native patients is essential to the doctors in China. Our study is such a large quantity investigation. Age at diagnosis and preoperative KPS score have been the welldocumented predictors of survival (Fazeny-Dorner et al., 2003; Mineo et al., 2007; Piroth et al., 2007; Gorlia et al., 2008). But some researchers have reported that patient age was not predictive of OS or PFR in small cohorts of the patients in clinical low-grade diffuse gliomas (Sabha et 
al., 2014). In our investigation, we have reaffirmed them in the study as independent factors for PFS and OS. KPS score change at 2 weeks after operation is also identified as a prognostic predictor. The relative risk is even lower in the multivariate analysis, which may be attributed to the influence of surgery complication.

Some researchers has reported that clinical variables (tumor diameter, extent of resection, performance status) was not predictive of OS or PFR in a small cohorts of the patients in clinical low-grade diffuse gliomas (Sabha et al., 2014).The study revealed that tumor maximal diameter was a radiological significant factor related to PFS and OS in the univariate analysis, not in the multivariate analysis. Single frontal lobe involvement was confirmed as a significant predictor of later progression and involvement of eloquent cortex or deep structure was independently associated with poor survival. All these indicate the site of the tumor may be more important than tumor size for the prognosis of these patients (Lutterbach et al., 2003).

$\mathrm{Ki}-67$ is a stable cell proliferation marker, which is only found in the active parts of the cell cycle: G1, G2, $\mathrm{S}$, and $\mathrm{M}$ phases. The latest WHO classification of central nervous system tumors includes $\mathrm{Ki}-67$ as an additional tool in histological typing and grading (Kleihues and Sobin, 2000). Our results showed Ki-67 were also a significant independent prognostic factor for GBM. Patients with low Ki-67 expression had much longer PFS and OS in comparison to those with high. Although some researches have suggested that PTEN and EGFR may influence the clinical outcomes of patients with glioma (Smith and Jenkins, 2000; Saito et al., 2006; Stojic et al., 2008), no correlation was found in the analysis.

Given the high specificity of the $1 \mathrm{p} 19 \mathrm{q}$ codeletion pattern, this clearly is a useful diagnostic marker. Yet, while $1 \mathrm{p} 19 \mathrm{q}$ codeletion cannot be considered a classical predictive marker insofar as it does not direct a specific tailored therapy, it has prognostic value, having repeatedly been shown to be associated with better response to chemotherapy and/or radiation, and thus a much longer survival., even after tumor recurrence. Some studies showed that $1 \mathrm{p} 19 \mathrm{q}$ codeletion is an early molecular change in the genesis of OD tumors, the presence of codeletion in a third of the GBMs with an OD component and absence in GBMs without an OD component entity (Singh VY et al., 2014). Several studies have identified that $1 p / 19 q$ deletion alone was predictive of OS but not PFR; 1p/19q codeletion, and PTEN deletion were predictive of OS ( $p=0.005, p=0.02$, respectively); both the interaction term of $1 \mathrm{p} / 19 \mathrm{q}$ and PTEN $(p<0.001)$ were found to be predictive of PFS (Sabha et al., 2014). Our study results showed that $1 \mathrm{p} 19 \mathrm{q}$ and PTEN was not a significant independent clinical prognostic factor related to PFS and OS for GBM. So, we thought losses of chromosomes $1 p$ and $19 q$ are correlated with diagnosis of higher chemosensitivity and better prognosis just in oligodendroglioma (Fontaine et al., 2008), not in GBM including oligodendrocyte component.

Matrix metalloproteinases (MMPs), the predominant component of the basement membrane, a family of zincdependent proteinases with extracellular matrix (ECM) remodeling and degrading properties, have been shown to be the key mediators in cancer metastasis. When released by tumor cells or the surrounding stroma, MMPs facilitate cancer cell invasion through the degradation of the ECM and basement membranes (VanMeter et al., 2001; Levicar et al., 2003; Rao, 2003; Yong, 2005). MMP9 are overexpressed also in many other tumors, such as in osteosarcomas (Jin et al., 2013), breast cancer (Darakhshan et al., 2013), cholangiocarcinoma (Kidoikhammouan et al., 2013), human fibrosarcoma cell (Yahayo et al., 2013), which all are overexpressed, suggesting a relevant role in tumor angiogenesis, growth, and invasion. MMP-9 has been found to be overexpressed in malignant glioma cells and may facilitate the invasiveness of tumor cells and tumor angiogenesis (Forsyth et al., 1999; Jadhav et al., 2004). Our results showed MMP9 was also a significant independent prognostic factor for GBM. Patients with low MMP9 expression had much longer PFS and OS compared with those high, which was in accordance with some researchers (Smith and Jenkins, 2000; Saito et al., 2006; Stojic et al., 2008).

Tumor resection can relieve the symptoms of the patients and provides conclusive pathologic diagnosis. Advances in neurosurgical techniques have improved the safety of resection. In our hospital., preoperative functional MRI and awake-mapping were routinely used for maximal resection while preserving key eloquent cortex if necessary. Only a quarter of patients achieved total resection, which was much lower than that reported by others in the country (Lee, 2008). Judging by postoperative enhancing MRI rather than by operator himself, may be the reason. Several studies have identified resection extent as an important predictor of clinical outcomes (Mineo et al., 2007; Piroth et al., 2007; Gorlia et al., 2008). In our study no correlation was found in the analysis. This was also not verified in some of the studies (Li et al., 2009; Sabha et al., 2014). But we still think total resection is a favorable prognostic factor, since the independent radiological prognostic factors found in the study relate closely to the tumor resection extent.

Postoperative radiotherapy has been recognized as chemotherapy based on alkylating agent has been without controversy. Several data showed temozolomide (TMZ) provided a statistically significant and clinically meaningful survival benefit, producing an increase in the median survival time from 12.1 to 14.6 months and in the two-year survival rate from $10 \%$ to $26 \%$ (Stupp et al., 2005). There are two kinds of patients receiving chemotherapy in our center. Patients with low O6-methylguanine-DNA methyltransferase (MGMT) expression would take TMZ only and those with high MGMT expression would receive combined chemotherapy of cisplatin (DDP) and teniposide (VM-26), DDP and TMZ or VM-26 and TMZ. The study confirmed adjuvant chemotherapy as an independent favorable prognostic factor for the clinical outcomes of GBM. Patients with adjuvant chemotherapy had a median PFS of 356 days and a median OS of 604 days, which were in accordance with the results from EORTC26981 and other researchers (Li et al., 2009).

Adjuvant chemotherapy could reduce residual tumor cells and prevent relapse, however, not all patients are suitable for adjuvant chemotherapy. Glioblastoma also show certain chemotherapy resistance. Screening 
Jun-Jie Qin et al

appropriate patients based on molecular markers for individualized adjuvant chemotherapy is necessary. The Topoisomerase II (Topo II) enzyme is a key target of anticancer agents because of the important role it plays in transcription regulation and chromatin remodeling. This nuclear enzyme is synthesized in late G1 and early $\mathrm{S}$-phase of the cell cycle, persists throughout the $\mathrm{G} 2$ and $\mathrm{M}$ phases and finally degrades as the cell enters G1, thereby identifying the percentage of cycling cells. Expression of this protein has been suggested to provide prognostic information in adult malignant gliomas (Holden et al., 1999; Taniguchi et al., 1999). In addition, TOPO II $\alpha$ has been related to a certain form of multidrug resistance to a number of anticancer agents in these neoplasms (Bredel et al., 2001). The findings of some studies indicated that topo II amplification may play a role in determining chemosensitivity of breast cancers to doxorubicin chemotherapy (Park et al., 2003). GST- $\pi$ expression in GBM was not a prognostic factor (Anda et al., 2003). Our results showed that Topo II and GST- $\pi$ was not significant independent prognostic factor for GBM.

Recently, there are many other treatments emerged to cure GBM. Glioblastoma also show certain radiation resistance, Residual tumor can use high dose hyper-fraction radiotherapy (HFRT), tumor interstitial radiotherapy and stereotactic radiosurgery. HFRT can increase the traditional radiation dose to 70.2 72Gy, which does not produce radioactive necrosis and can enhance the ability of inhibiting tumor recurrence. Stereotactic implanted (interstitial radiotherapy) 125 I plasmid cooperate with HFRT can significantly improve the effect of treatment, which is superior to the traditional combination of radiotherapy and chemotherapy (Smrdel et al., 2014). For sensitive rate of chemotherapy about glioblastoma is different from $40 \%$ to $80 \%$, recently there are also many report about immunotherapy, genetherapy of glioblastoma, but the effect are not really sure because of the ununified evaluation standard of the treatment method and effect, only as a part of comprehensive treatment, not a substitute of radiotherapy and chemotherapy.

In conclusion, this is a valuable retrospective study with a large number of GBM patients and full-scale analysis. The results suggest that analysis of prognostic markers in glioblastoma multiforme is complex. Age at diagnosis, preoperative KPS score, KPS score change at 2 weeks postoperation, involvement of brain lobe, involvement of eloquent cortex or deep structure, $\mathrm{Ki}$ 67and MMP9 expression level and adjuvant chemotherapy correlate significantly with the prognosis of these patients with GBM. 1p19q deletion had no correlate significantly with the prognosis of these patients with GBM.

In the future, the most appropriate new treatment might be chosen and administered alone or in combination (e.g. theoretically bevacizumab for GBMs highly expressing VEGF or Erlotinib for those with EGFR amplification). On the other hand, many treatments, such as immunotherapy and gene therapy, might be improved, as a substitute of radiotherapy and chemotherapy.

\section{Acknowledgements}

This work was supported by grants from the "Capital Health Project" (Z131100006813011), the "basic-clinical scientific research cooperation foundation of the capital medical university" (13JL04), "National Key Technology Research and Development Program of the Ministry of Science and Technology of China" (2013BAI09B03), and "Beijing Institute for Brain Disorders" (BIBDPXM2013_014226_07_000084).

\section{References}

Anda T, Shabani HK, Tsunoda K, et al (2003). Relationship between expression of O6-methylguanine-DNA methyltransferase, glutathione-S-transferase pi in glioblastoma and the survival of the patients treated with nimustine hydrochloride: an immunohistochemical analysis. Neurol Res, 25, 241-8.

Bredel M (2001). Anticancer drug resistance in primary human brain tumors. Brain Res Rev, 35, 161-204.

Chen SD, Song MM, Zhong ZQ et al (2012). Knockdown of radixin by RNA interference suppresses the growth of human pancreatic cancer cells in vitro and in vivo. Asian Pac J Cancer Prev, 13, 753-9.

Cheng CL, Johnson SP, Keir ST, et al (2005). Poly(ADP-ribose) polymerase-1 inhibition reverses temozolomide resistance in a DNA mismatch repair-deficient malignant glioma xenograft. Mol Cancer Ther, 4, 1364-8.

Darakhshan S, Bidmeshkipour A, Khazaei M, et al (2013). Synergistic effects of tamoxifen and tranilast on VEGF and MMP-9 regulation in cultured human breast cancer cells. Asian Pac J Cancer Prev, 14, 6869-74.

Fazeny-Dorner B, Wenzel C, et al (2003). Survival and prognostic factors of patients with unresectable glioblastoma multiforme. Anticancer Drugs, 14, 305-12.

Fontaine D, Vandenbos F, et al (2008). Diagnostic and prognostic values of $1 p$ and $19 q$ deletions in adult gliomas: critical review of the literature and implications in daily clinical practice. Rev Neurol (Paris), 164, 595-604.

Forsyth PA, Wong H, Laing TD, et al (1999). Gelatinase-A (MMP-2), gelatinase-B (MMP-9) and membrane type matrix metalloproteinase-1 (MT1-MMP) are involved in different aspects of the pathophysiology of malignant gliomas. $\mathrm{Br} \mathrm{J}$ Cancer, 79, 1828-35.

Gorlia T, van den Bent MJ, Hegi ME, et al (2008). Nomograms for predicting survival of patients with newly diagnosed glioblastoma: prognostic factor analysis of EORTC and NCIC trial 26981-22981/CE.3. Lancet Oncol, 9, 29-38.

Hoffman S, Propp JM, McCarthy BJ (2006). Temporal trends in incidence of primary brain tumors in the United States, 1985-1999. Neuro Oncol, 8, 27-37.

Holden JA, Townsend JJ (1999). DNA topoisomerase II-alpha as a proliferation marker in astrocytic neoplasms of the central nervous system: correlation with MIB1 expression and patient survival. Mod Pathol, 12, 1094-100.

Jadhav U, Chigurupati S, Lakka SS, Mohanam S (2004). Inhibition of matrix metalloproteinase- 9 reduces in vitro invasion and angiogenesis in human microvascular endothelial cells. Int J Oncol, 25, 1407-14.

Jeremic B, Milicic B, Grujicic D, Dagovic A, Aleksandrovic J (2003). Multivariate analysis of clinical prognostic factors in patients with glioblastoma multiforme treated with a combined modality approach. J Cancer Res Clin Oncol, 129, 477-84.

Jin J, Cai L, Liu ZM, et al (2013). miRNA-218 inhibits osteosarcoma cell migration and invasion by downregulating of TIAM1, MMP2 and MMP9. Asian Pac J 
Cancer Prev, 14, 3681-4.

Prognostic Factors Influencing Clinical Outcomes of Malignant Glioblastoma Multiforme

Kang SG, Kim JH, Nam DH, Park K (2005). Clinical and radiological prognostic factors of anaplastic oligodendroglioma treated by combined therapy. Neurol Med Chir, 45, 232-8; discussion 238-9.

Kidoikhammouan S, Seubwai W, Tantapotinan N, et al (2013). TNP-470, a methionine aminopeptidase-2 inhibitor, inhibits cell proliferation, migration and invasion of human cholangiocarcinoma cells in vitro. Asian Pac J Cancer Prev, 13, 155-60.

Kjaergaard J, Wang LX, Kuriyama H, Shu S, Plautz GE (2005). Active immunotherapy for advanced intracranial murine tumors by using dendritic cell-tumor cell fusion vaccines. $J$ Neurosurg, 103, 156-64.

Kleihues P, Sobin LH (2000). World Health Organization classification of tumors. Cancer, $\mathbf{8 8}, 2887$.

Lee SD (2008). Notice of retraction of "The experience with Ritleng intubation system in patients with congenital nasolacrimal duct obstruction". 67, 344-8. J Chin Med Assoc, 71, 329.

Levicar N, Nuttall RK, Lah TT (2003). Proteases in brain tumour progression. Acta Neurochir (Wien), 145, 825-38.

Li S, Jiang T, Wang Z (2008). Impact of p53 status to response of temozolomide in low MGMT expression glioblastomas: preliminary results. Neurol Res, 30, 567-70.

Li SW, Qiu XG, Chen BS, et al (2009). Prognostic factors influencing clinical outcomes of glioblastoma multiforme. Chin Med J, 122, 1245-9.

Louis DN, Ohgaki H, Wiestler OD, et al (2007). The 2007 WHO classification of tumours of the central nervous system. Acta Neuropathol, 114, 97-109.

Lutterbach J, Sauerbrei W, Guttenberger R (2003). Multivariate analysis of prognostic factors in patients with glioblastoma. Strahlenther Onkol, 179, 8-15.

Macdonald DR, Cascino TL, Schold SC Jr, Cairncross JG (1990). Response criteria for phase II studies of supratentorial malignant glioma. J Clin Oncol, 8, 1277-80.

Mineo JF, Bordron A, Baroncini M, et al (2007). Prognosis factors of survival time in patients with glioblastoma multiforme: a multivariate analysis of 340 patients. Acta Neurochir, 149, 245-52; discussion 252-43.

$\mathrm{Ng}$, SS, Cheung YT, An XM, et al (2007). Cell cycle-related kinase: a novel candidate oncogene in human glioblastoma. J Natl Cancer Inst, 99, 936-48.

Park K, Kim J, Lim S, et al (2003).Topoisomerase II-alpha (topo II) and HER2 amplification in breast cancers and response to preoperative doxorubicin chemotherapy. Eur J Cancer, 39, 631-4.

Piroth MD, Gagel B, Pinkawa M, et al (2007). Postoperative radiotherapy of glioblastoma multiforme: analysis and critical assessment of different treatment strategies and predictive factors. Strahlenther Onkol, 183, 695-702.

Rao JS (2003). Molecular mechanisms of glioma invasiveness: the role of proteases. Nat Rev Cancer, 3, 489-501.

Sabha N, Knobbe CB, Maganti M, et al (2014). Analysis of IDH mutation, $1 \mathrm{p} / 19 \mathrm{q}$ deletion, and PTEN loss delineates prognosis in clinical low-grade diffuse gliomas. Neuro Oncol, 16, 914-923.

Saito T, Hama S, Kajiwara Y, et al (2006). Prognosis of cerebellar glioblastomas: correlation between prognosis and immunoreactivity for epidermal growth factor receptor compared with supratentorial glioblastomas. Anticancer Res, 26, 1351-7.

Sathornsumetee S, Rich JN (2006). New treatment strategies for malignant gliomas. Expert Rev Anticancer Ther, 6, 1087-104.

Schneider T, Mawrin C, Scherlach C, Skalej M, Firsching R
(2010). Gliomas in adults. Dtsch Arztebl Int, 107, 799-807.

Singh VY, Chacko G, Chacko AG, et al (2014). Fluorescence in situ hybridization for $1 \mathrm{p}, 19 \mathrm{q}$ status in a cohort of glial neoplasms. Neurol India, 62, 32-6.

Smith JS, Jenkins RB (2000). Genetic alterations in adult diffuse glioma: occurrence, significance, and prognostic implications. Front Biosci, 5, 213-31.

Smrdel U, Kovac V, Popovic M, Zwitter M (2014). Glioblastoma patients in Slovenia from 1997 to 2008. Radiol Oncol, 48, 72-9.

Stojic J, Hagemann C, Haas S, et al (2008). Expression of matrix metalloproteinases MMP-1, MMP-11 and MMP-19 is correlated with the WHO-grading of human malignant gliomas. Neurosci Res, 60, 40-9.

Stupp R, Hegi ME, van den Bent MJ, et al (2006). Changing paradigms--an update on the multidisciplinary management of malignant glioma. Oncologist, 11, 165-80.

Stupp R, WP Mason, van den Bent MJ, et al (2005). Radiotherapy plus concomitant and adjuvant temozolomide for glioblastoma. $N$ Engl J Med, 352, 987-96.

Stupp R, Pavlidis N, Jelic S (2005). ESMO minimum clinical recommendations for diagnosis, treatment and follow-up of malignant glioma. Ann Oncol, 16, 64-5.

Stupp R, Weber DC (2005). The role of radio-and chemotherapy in glioblastoma. Onkologie, 28, 315-7.

Taniguchi K, Wakabayashi T, Yoshida T, et al (1999). Immunohistochemical staining of DNA topoisomerase II alpha in human gliomas. $J$ Neurosurg, 91, 477-82.

Tramacere F, Gianicolo E, Serinelli M, et al (2008). Multivariate analysis of prognostic factors and survival in patients with "glioblastoma multiforme. Clin Ter, 159, 233-8.

VanMeter TE, Rooprai HK, Kibble MM, et al (2001). The role of matrix metalloproteinase genes in glioma invasion: codependent and interactive proteolysis. $J$ Neurooncol, $\mathbf{5 3}$, 213-35.

Wen PY, Yung WK, Lamborn KR, et al (2006). Phase I/II study of imatinib mesylate for recurrent malignant gliomas: North American Brain Tumor Consortium Study 99-08. Clin Cancer Res, 12, 4899-907.

Yahayo W, Supabpho A, Supabphol R (2013). Suppression of human fibrosarcoma cell metastasis by Phyllanthus emblica extract in vitro. Asian Pac J Cancer Prev, 14, 6863-7.

Yong VW (2005). Metalloproteinases: mediators of pathology and regeneration in the CNS. Nat Rev Neurosci, 6, 931-44. 